\title{
Adult Partial Anomalous Pulmonary Venous Connection With Drainage to Left Atrium and Inferior Vena Cava Clearly Visualized on a Combination of Multiple Imaging Techniques
}

Hajime Kasai, MD, PhD; Toshihiko Sugiura, MD, PhD; Takahiro Arano;

Hiroki Shoji, MD; Takayuki Jujo, MD, PhD; Seiichiro Sakao, MD, PhD;

Nobuhiro Tanabe, MD, PhD; Koichiro Tatsumi, MD, PhD

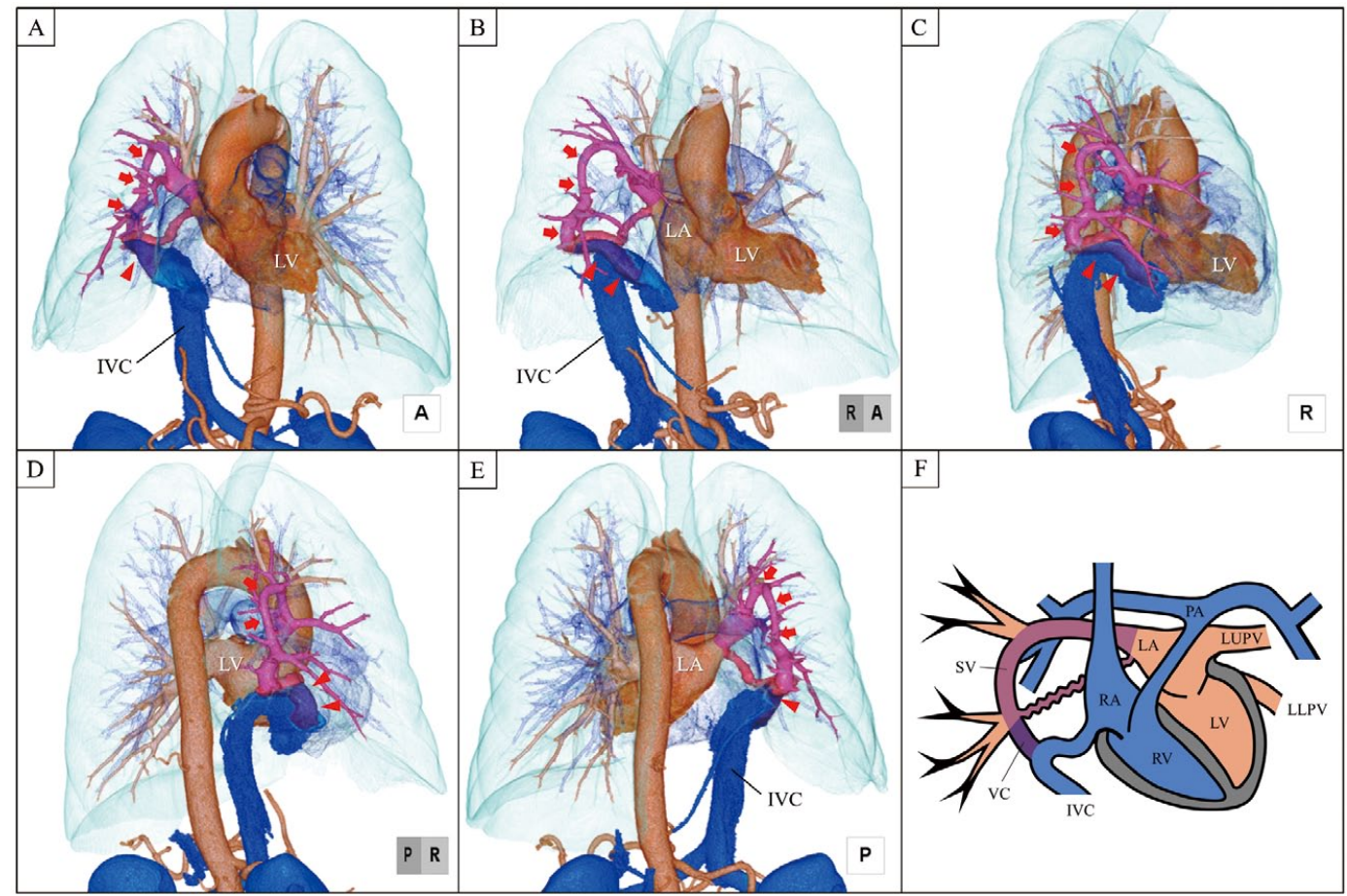

Figure 1. Contrast-enhanced computed tomography (CT) of the chest showing tortuous right pulmonary vein (arrows) connected to the left atrium (LA) and the inferior vena cava (IVC) via the abnormal venous connection (arrowheads) on (A) anterior, (B) right anterior oblique, (C) right side, (D) right posterior oblique, and (E) posterior views. (F) Schematic diagram based on CT. LLPV, left lower pulmonary vein; LUPV, left upper pulmonary vein; LV, left ventricle; PA, pulmonary artery; RA, right atrium; RV, right ventricle; $\mathrm{SV}$, scimitar vein; VC, venous connection.

$\mathbf{P}$ artial anomalous pulmonary venous connection (PAPVC) is a rare congenital cardiac defect in which abnormal connections exist between 1 to all of the PV and the right atrium (RA) or vena cava. Rarely,
PAPVC involves connection of the right lower PV to the inferior vena cava (IVC) with an additional drainage to the left atrium (LA). ${ }^{1}$ The diagnosis and evaluation of PAPVC require imaging using various modalities, such as echo-

Received January 12, 2017; revised manuscript received February 14, 2017; accepted February 28, 2017; released online March 31, 2017 Time for primary review: 5 days

Department of Respirology, Graduate School of Medicine (H.K., T.S., H.S., T.J., S.S., N.T., K.T.), Department of Medicine, School of Medicine (T.A.), Department of Advanced Medicine in Pulmonary Hypertension, Graduate School of Medicine (T.J., N.T.), Chiba University, Chiba, Japan

Mailing address: Hajime Kasai, MD, PhD, Department of Respirology, Graduate School of Medicine, Chiba University, 1-8-1 Inohana, Chuo-ku, Chiba 260-8670, Japan. E-mail: daikasai6075@yahoo.co.jp

ISSN-1346-9843 All rights are reserved to the Japanese Circulation Society. For permissions, please e-mail: cj@j-circ.or.jp 


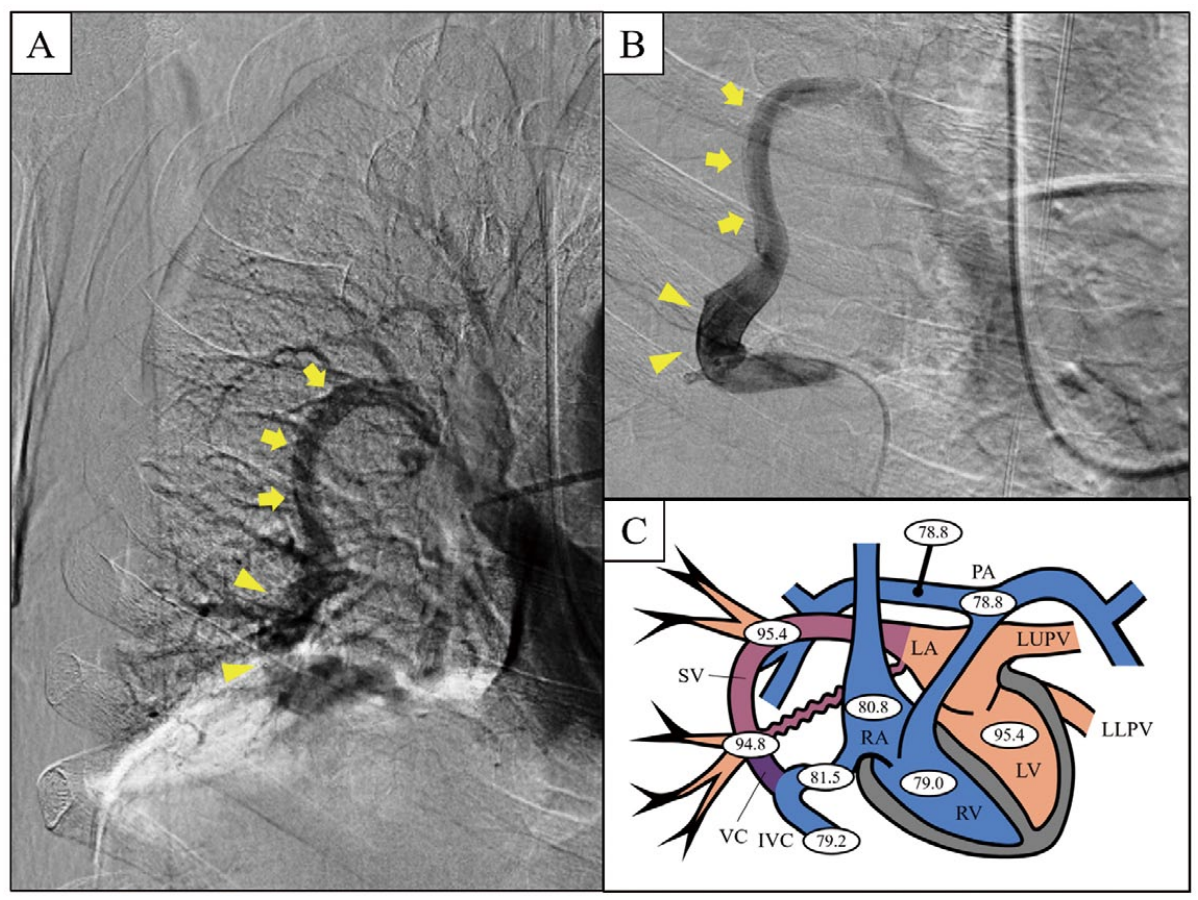

Figure 2. Angiography of the (A) right pulmonary artery and (B) inferior vena cava (IVC) showing a curved vessel similar to the shape of a scimitar (arrows) connecting to the left atrium (LA) and IVC via the abnormal venous connection (arrowheads), similar to 3-D computed tomography. (C) Oximetry indicated a small step-up in percent saturation in the upper part of the inferior vena cava IVC. Data given as percentages (\%). LLPV, left lower pulmonary vein; LUPV, left upper pulmonary vein; LV, left ventricle; PA, pulmonary artery; RA, right atrium; RV, right ventricle; SV, scimitar vein; VC, venous connection.

cardiography, computed tomography (CT), magnetic resonance imaging, and cardiac catheterization. ${ }^{2}$ It is sometimes difficult, however, to sufficiently grasp the morphology and hemodynamics of PAPVC. Here we present the case of a patient with PAPVC with dual drainage to the LA and IVC, which was diagnosed on the combination of multiple imaging modalities.

A 58-year-old man was admitted to hospital because of abnormal lung shadow detected during routine health check-up. He had a history of surgery for diaphragmatic hernia at 5 years of age. On admission, he was asymptomatic, with $99 \%$ oxygen concentration in room air on pulse oximetry. Chest X-ray showed elevation of the right diaphragm and a curved-vessel shadow similar to the shape of a scimitar (Figure S1A). Contrast-enhanced CT of the chest, maximum intensity projection constructed from it, and 3-D image reconstruction (3D-CT) showed 2 anomalous PV connections, a scimitar-like vein (SV) connecting the right upper and lower PV, and an anomalous venous connection (VC) between the right lower PV and IVC (Figures 1,S1B,C). Echocardiography showed no signs of right-side dysfunction or congenital heart disease. The grade of tricuspid regurgitation (TR) was trivial, and the TR pressure gradient was $20.5 \mathrm{mmHg}$. Furthermore, although visualization on echocardiography was poor, blood flow in the VC connected to the IVC was both right to left and left to right. Right heart catheterization (RHC) showed a pulmonary artery pressure (PAP) of $24 / 13 \mathrm{mmHg}$ (mean, $18 \mathrm{mmHg}$ ), cardiac output $(\mathrm{CO})$ of $7.6 \mathrm{~L} / \mathrm{min}$ on thermodilution, and pulmonary : systemic blood flow ratio
(Qp/Qs) 0.9. Shunt rates were 7.4\% and 9.6\% as estimated on RHC and pulmonary perfusion scintigraphy, respectively. On RHC oximetry, percent saturation in the lower IVC, upper IVC, and right atrium were $79.2 \%, 81.5 \%$, and $80.8 \%$, respectively, with a small step-up in the upper part of the IVC (Figure 2C). On balloon occlusion of the VC, PAP, and CO changed to $24 / 5 \mathrm{mmHg}$ (mean, $13 \mathrm{mmHg}$ ) and $6.2 \mathrm{~L} / \mathrm{min}$, respectively, although the LA pressure did not change. In addition, oxygen saturation of the right and left PA decreased from $78.8 \%$ to $75.4 \%$ in the right PA and to $74.6 \%$ in the left PA. The pulmonary angiography findings were similar to those of 3D-CT (Figure 2A,B). Furthermore, blood flow from the VC to the RA through the IVC was small on VC angiography (Movie S1). Thus, we diagnosed PAPVC with dual drainage to the LA and IVC. There were no indications for surgery and transcatheter repair, therefore careful observation was continued.

PAPVC is detected in $0.1-0.2 \%$ of adults on routine CT..$^{3,4}$ To the best of our knowledge, 18 cases of PAPVC with dual drainage treated by transcatheter repair have been reported in the literature. ${ }^{5}$ It is assumed that PAPVC with dual drainage is a rare subset of PAPVC, but the incidence of PAPVC involving duplicate anomalous $\mathrm{VC}$ is difficult to estimate because patients are often asymptomatic. $5^{-1}$

It is difficult to interpret the morphology of PAPVC on planar CT because PAPVC presents many variations. ${ }^{\mathbf{6}}, \mathbf{7}$ $\mathrm{PV}$ angiography can provide anatomical information, but the procedure is invasive. 3D-CT is a useful method for physicians to understand the complicated anatomical 
structure of PAPVC because it provides more information to facilitate an appropriate cardiac catheterization approach.

In the present case, it was possible to clearly delineate SV and VC on 320-row multidetector CT, although its morphology was strongly meandering and complicated (Figure 1F). The blood flow inside the VC was small and involved to-and-fro flow, as evident from RHC oximetry and echocardiography. Here we have presented the case of a patient with PAPVC with dual drainage to the LA and IVC, in whom the morphology of abnormal vessels and hemodynamics were clearly delineated using multiple imaging techniques. In particular, 3D-CT was a helpful method for understanding the anatomy of PAPVC with dual drainage.

\section{References}

1. Fuchigami T, Gabe A, Takahashi K, Nishioka M, Akashige T, Nagata N. Thoracoscopic surgery for partial anomalous pulmonary venous connection with dual drainage. J Card Surg 2015; 30: $764-766$.

2. Recto MR, Sadlo H, Sobczyk WL. Rare case of persistent left superior vena cava to left upper pulmonary vein: Pathway for paradoxical embolization and development of transient ischemic attack and subsequent occlusion with an Amplatzer vascular plug. J Invasive Cardiol 2007; 19: E313-E316.

3. Ho ML, Bhalla S, Bierhals A, Gutierrez F. MDCT of partial anomalous pulmonary venous return (PAPVR) in adults. $J$ Thorac
Imaging 2009; 24: 89-95.

4. Haramati LB, Moche IE, Rivera VT, Patel PV, Heyneman L, McAdams HP, et al. Computed tomography of partial anomalous pulmonary venous connection in adults. J Comput Assist Tomogr 2003; 27: 743-749.

5. Luciano D, Laux D, Boudjemline Y, Hascoet S, Lusson JR, Sorensen C, et al. Transcatheter therapy in partially abnormal pulmonary venous return with additional drainage to the left atrium. Int J Cardiol 2013; 170: 221-226.

6. Wilson W, Horlick E, Benson L. Successful transcatheter occlusion of an anomalous pulmonary vein with dual drainage to the left atrium. Catheter Cardiovasc Interv 2015; 85: 1212-1216.

7. Jujo T, Tanabe N, Sugiura T, Naito A, Shigeta A, KitazonoSaitoh M, et al. Importance of carefully interpreting computed tomography images to detect partial anomalous pulmonary venous return. Respir Investig 2016; 54: 69-74.

\section{Supplementary Files}

\section{Supplementary File 1}

Figure S1. (A) Chest X-ray showing elevation of the right diaphragm and a curved-vessel shadow similar to the shape of a scimitar (arrows)

\section{Supplementary File 2}

Movie S1. Angiography of venous connection (VC) showing low blood flow from VC to the right atrium through the inferior vena cava.

Please find supplementary file(s);

http://dx.doi.org/10.1253/circj.CJ-17-0029 